\title{
Organic Solar Cells Using Nanoimprinted Transparent Metal Electrodes**
}

\author{
By Myung-Gyu Kang, Myung-Su Kim, Jinsang Kim,* and L. Jay Guo*
}

Cost effective and highly efficient renewable energy is becoming ever more important in our age of rising energy prices and global climate change. Solar energy is a nonexhaustible and green energy. Organic solar cells (OSC) have the merits of low cost and simplistic fabrication in addition to compatibility with flexible plastic substrates over large areas. They have therefore been considered a promising energy conversion platform for clean and carbon-neutral energy production. ${ }^{[1,2]}$ In recent years, the power conversion efficiency of OSCs based on conjugated polymers has steadily increased through improved energy harvesting, enhanced exciton separation in improved device structures, ${ }^{[3]}$ and optimization of processing parameters, e.g., solvent evaporation time, ${ }^{[4]}$ and annealing conditions. ${ }^{[5,6]}$ Most OSCs are built on indium tin oxide (ITO) coated substrates because ITO offers transparency in the visible range of the electromagnetic spectrum as well as good electrical conductivity. However, ITO is not the optimum electrode for solar cell applications as it has been reported that the band structure of ITO hinders efficient photocurrent generation. ${ }^{[7]}$ Moreover, the poor mechanical stability of ITO can cause device failure when an ITO-coated flexible substrate is bent. ${ }^{[8]}$ In addition, the limited supply of indium and the increasing demand from the rapidly expanding display market have increased the cost of ITO drastically, which potentially prevents the realization of low cost and large scale OSC fabrication. Therefore, there is a strong need to find alternative materials that can replace ITO as high transparency electrode. Some examples that have been investigated recently are nanotube networks, ${ }^{[9-12]}$ and Ag wire grids. ${ }^{[13,14]}$

In this communication, we report on high transparency metal wire grid electrodes for organic solar cell applications. The high transparency metal electrodes are fabricated by nanoimprint lithography (NIL) ${ }^{[15]}$ and have several advan-

[*] Prof. L. J. Guo, M.-G. Kang

Department of Electrical Engineering and Computer Science, The University of Michigan

1301 Beal Ave., Ann Arbor, MI48109 (USA)

E-mail: guo@eecs.umich.edu

Prof. J. Kim, M.-S. Kim

Department of Materials Science and Engineering, Chemical

Engineering, Macromolecular Science and Engineering,

The University of Michigan

2300 Hayward St., Ann Arbor, MI 48109 (USA)

E-mail: jinsang@umich.edu

[**] This work was supported in part by NSF and KACST. The authors thank Prof. Max Shtein for allowing us to use the metal evaporator for organic solar cell fabrication. This article was amended for print publication. tages over other highly transparent electrodes including ITO. Firstly, the work function of nanopatterned transparent metal electrodes can be easily tuned by choosing different metal materials, which allows systematic studies of the effect that the electrode work function has on the device performance. Secondly, a high electrical conductivity can be achieved without seriously compromising the transparency. ${ }^{[16]}$ Thirdly, the light absorption and the resulting power conversion efficiency of OSCs can possibly be increased by means of light trapping phenomena that are induced by the grating structure. ${ }^{[17-20]}$ Lastly, large area organic solar cells with transparent metal electrodes could be realized at a low cost by using a newly developed roll-to-roll nanoimprint technique. ${ }^{\text {[21] }}$ Herein, a simple fabrication method which is readily adapted to roll-to-roll processing for high throughput and low cost is shown. We demonstrate that the power conversion efficiency of OSCs that incorporate nanopatterned metal electrodes (e.g., inexpensive $\mathrm{Cu}$ ) is comparable to that of analogous cells using high performance ITO electrodes. We also show the effect of the anode work function on the OSC's fill factor (FF) as well as the photocurrent. A simple design principle to make suitable transparent metal electrodes is discussed.

The mold for the NIL production of the transparent metal electrodes consists of two sets of grating structures which are orthogonally oriented to each other. The major grating with a $700 \mathrm{~nm}$ period determines the overall optical transparency; specifically, the transmittance of the metal electrode is determined by the line-width of the grating lines, or equivalently, the opening ratio of the grating. The orthogonal secondary grating with a $10 \mu \mathrm{m}$ period and a $400 \mathrm{~nm}$ line-width is used to ensure electrical connectivity of the major $700 \mathrm{~nm}$ period grating in case of a fabrication defect. In order to achieve high optical transparency, the line-width of the original $700 \mathrm{~nm}$ period grating with a $50 \%$ duty cycle was reduced by a simple wet-chemical etching process rather than the shadow evaporation method used in our previous work. ${ }^{[16]}$ The wet etching approach produced gratings with smoother sidewalls, which could reduce surface scattering of electrons and therefore increase the conductivity of the wire electrode. Figure 1a shows the scanning electron microscopy (SEM) image of the original $\mathrm{SiO}_{2}$ grating with a $700 \mathrm{~nm}$ period and a $50 \%$ duty cycle, and Figure $1 \mathrm{~b}$ the SEM image after wet etching using a buffered $\mathrm{HF}$ solution for $80 \mathrm{~s}$. A line-width of $70 \mathrm{~nm}$ was achieved by using this technique.

The rectangular grid pattern of the imprinting mold (Fig. 2a) was then created by performing NIL twice using two grating molds with an orientation orthogonal to one another. NIL was 


\section{ADVANCED MATERIALS}

a)

b)
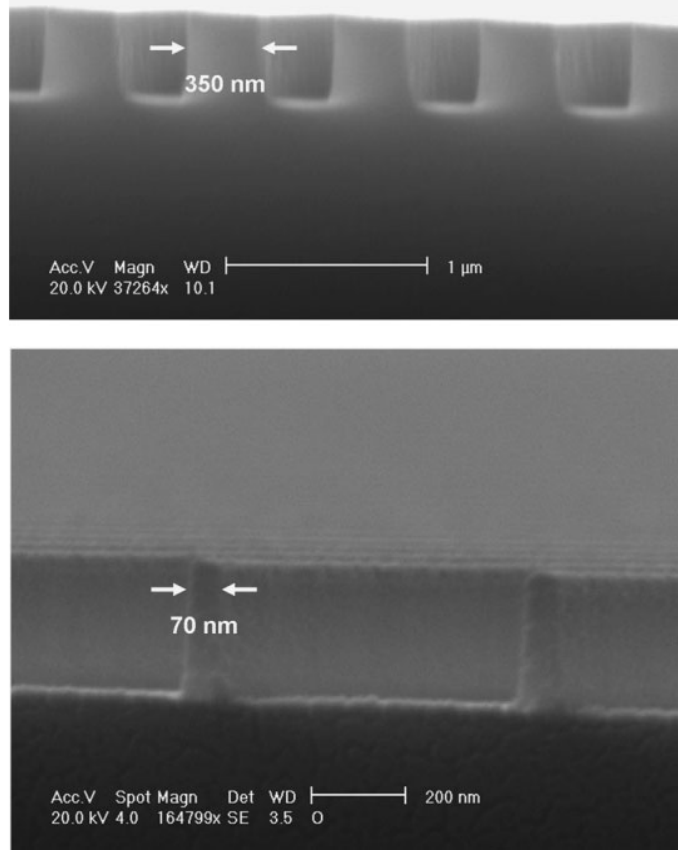

Figure 1. a) Scanning electron microscopy (SEM) image of the original $\mathrm{SiO}_{2}$ grating with a period of $700 \mathrm{~nm}$ and a duty cycle of $50 \%$; b) SEM image after wet etching with buffered HF solution (Buffered HF improved) for $80 \mathrm{~s}$.

then used again to transfer the rectangular patterns of the mold to a resist layer on a glass substrate. Standard oxygen residual etching, metallization, and lift-off completed the fabrication of the transparent metal electrodes on glass. Mold fabrication is described in detail in the Experimental section. Figure $2 b$ shows the SEM image of an as-fabricated metal electrode on a glass substrate. Typical samples used in this study had a patterned transparent metal electrode area of $2 \times 2 \mathrm{~cm}^{2}$.

$\mathrm{Au}, \mathrm{Cu}$, and $\mathrm{Ag}$ electrodes with a thickness of $40 \mathrm{~nm}$ were prepared using the method described above to show that the fabrication process can be readily applied to various metals; and to study the effect of the anode's work function on the cell performance, e.g., the photocurrent, the fill factor (FF), and the open circuit voltage $\left(V_{\mathrm{oc}}\right)$. The work functions for $\mathrm{Au}, \mathrm{Cu}$, and $\mathrm{Ag}$ are $5.22 \mathrm{eV}, 4.65 \mathrm{eV}$, and $4.0 \mathrm{eV}$, respectively. ${ }^{[22]}$ Optical transmittance of as-prepared transparent $\mathrm{Au}, \mathrm{Cu}$, and $\mathrm{Ag}$ electrodes was measured in the visible wavelength range and is shown in Figure 3. For comparison, the optical transmittance of a commercial high quality ITO used for the control device was also measured and included in the figure. All transmittance measurements were referenced to air.

ITO has a peak transmittance of $90 \%$ at $500 \mathrm{~nm}$ and an average transmittance of $87 \%$ over the entire visible range. The transmittance of the nanopatterned metal electrodes is relatively flat in the visible region. This means that the a)

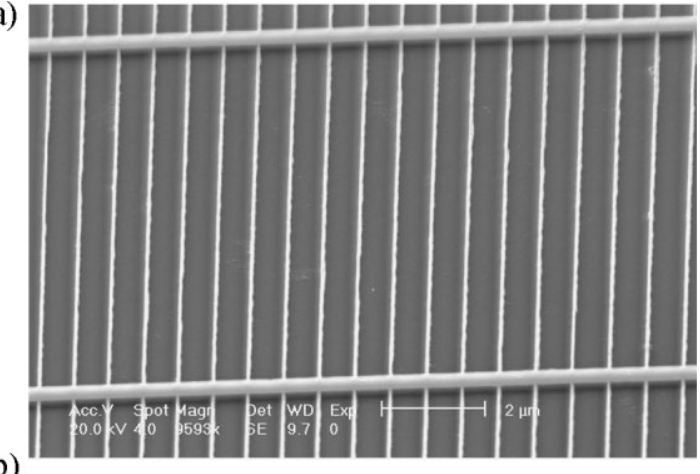

b)

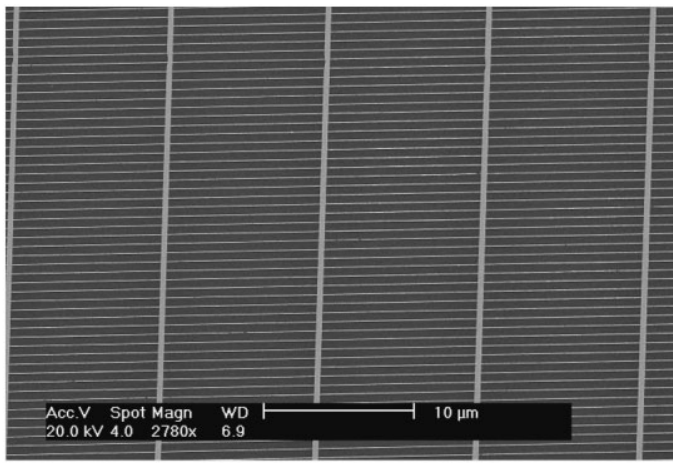

c)

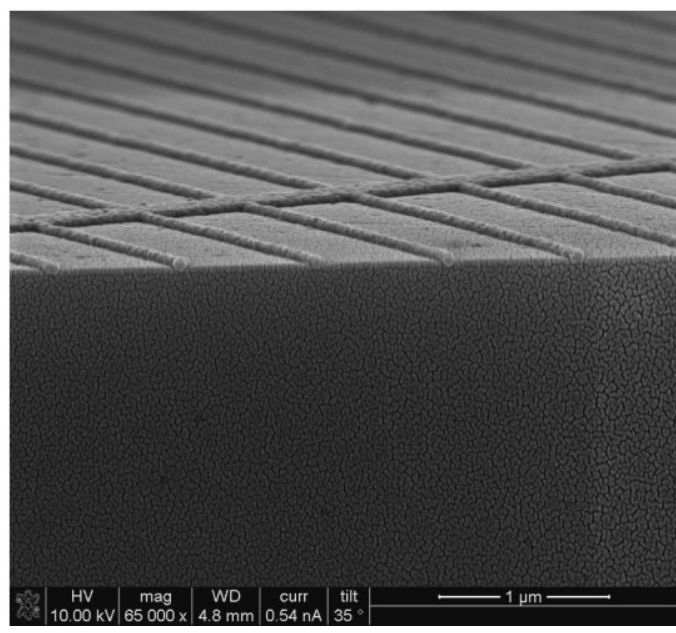

Figure 2. a) SEM image of the nanoimprint lithography (NIL) mold with a rectangular grid pattern created by applying NIL twice using a $90^{\circ}$ offset; b) SEM image of an as-fabricated metal electrode on glass; c) Perspective image of $b$ ).

wavelength dependency observed previously for nanoimprinted metal electrodes with wider line-widths (e.g., $200 \mathrm{~nm}$ and $120 \mathrm{~nm}),{ }^{[16]}$ was significantly reduced by using a narrower metal line-width (i.e., $70 \mathrm{~nm}$ ). The average transmittance in the visible range is $84 \%, 83 \%$, and $78 \%$ for the $\mathrm{Au}, \mathrm{Cu}$, and $\mathrm{Ag}$ electrode, respectively. Interestingly, the $\mathrm{Ag}$ electrode has a lower transmittance than the $\mathrm{Au}$ and $\mathrm{Cu}$ electrodes even though it has the same line-width and thickness, which may be related to the dispersion properties of Ag. The sheet resistance, another important parameter of transparent and conductive electrodes, was measured using the four-point probe method. 


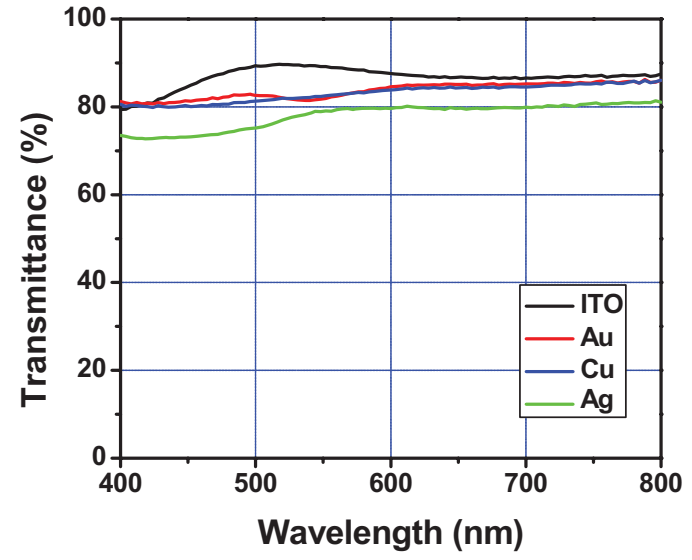

Figure 3. Optical transmittance of the nanoimprinted transparent $\mathrm{Au}, \mathrm{Cu}$, and $\mathrm{Ag}$ electrodes and a conventional high performance indium tin oxide (ITO) electrode in the visible range.

High performance ITO showed a sheet resistance of $12 \Omega \cdot \mathrm{sq}^{-1}$, while the $\mathrm{Au}, \mathrm{Cu}$, and $\mathrm{Ag}$ electrodes exhibited higher sheet resistances of $24 \Omega \cdot \mathrm{sq}^{-1}, 28 \Omega \cdot \mathrm{sq}^{-1}$, and $23 \Omega \cdot \mathrm{sq}^{-1}$, respectively. This increased sheet resistance is due to the reduction in line-width, but can be easily compensated by depositing thicker metal layers. For example, a $80 \mathrm{~nm}$ thick $\mathrm{Au}$ electrode has a by a factor of three reduced sheet resistance $\left(8 \Omega \cdot \mathrm{sq}^{-1}\right)$ at the cost of a small decrease $(7 \%)$ in optical transmittance only. Therefore, high transparency metal wire grid electrodes are a promising alternative to ITO because through these nanostructured electrodes, factors such as transmittance and sheet resistance can be easily tuned by variation of metal line-width and thickness. Moreover, if the structures can be fabricated using a roll-to-roll process, the cost effective and large area fabrication of nanopatterned electrodes can be realized. In order to demonstrate the feasibility of this, we fabricated a transparent metal electrode onto a poly(3,4-ethylenedioxythiophene)/poly(styrene sulfonic acid) (PEDOT:PSS)-coated glass substrate using a flexible poly(dimethylsiloxane) (PMDS) mold and a metal transfer process. Flexible molds are required for the rollto-roll nanoimprint process. ${ }^{[21]}$ The soft PDMS stamp was first fabricated from a nanoimprinted resist template, ${ }^{[23,24]}$ followed by deposition of a $40 \mathrm{~nm}$-thick $\mathrm{Cu}$ layer. After spin-casting a PEDOT:PSS layer on a glass substrate, the PDMS stamp was laminated on top of the PEDOT:PSS layer and briefly baked. Peeling the PDMS stamp from the PEDOT:PSS layer left behind the patterned $\mathrm{Cu}$ electrodes on the PEDOT:PSS layer (Fig. 4a). Detailed fabrication conditions are provided in the Experimental section. Figure $4 b$ shows the SEM image of a PDMS stamp coated with $40 \mathrm{~nm} \mathrm{Cu}$ on top, and Fig. $4 \mathrm{c}$ shows the SEM image of the transferred $\mathrm{Cu}$ electrode on top of the PEDOT:PSS layer. These results imply that the fabrication of nanopatterned metal electrodes can be extended to roll-to-roll processing.

In order to evaluate the potential use of nanopatterned metal electrodes as highly transparent, conducting electrodes in organic optoelectronic devices, bulk heterojunction devices were fabricated and compared with those fabricated from high quality ITO. $40 \mathrm{~nm}$ thick, wire-grid patterned $\mathrm{Au}, \mathrm{Cu}$, and $\mathrm{Ag}$ electrodes were chosen as anode materials. The multi-layer solar cells comprised ITO or nanopatterned metal anode, conducting PEDOT:PSS layer, light absorbing P3HT:PCBM blend (1:1 wt/wt), and $\mathrm{LiF} / \mathrm{Al}$ cathode (from bottom to top). Fabrication details are given in the Experimental section. We would like to point out that because the nanopatterned metal electrodes were of uniform thickness, we were able to spin coat very thin PEDOT:PSS layers $(\sim 50 \mathrm{~nm})$ on top of them without causing current shunt paths which would lower the fill factor and therefore reduce the power efficiency. In the case of random nano-wire structures such as the Ag nano-wire mesh used in previous studies, ${ }^{[13,14]}$ a thin coating of PEDOT layer could increase the current shunt path due to the roughness or unevenness of the random $\mathrm{Ag}$ wires.

The fabricated solar cells had an area of approximately $0.1 \mathrm{~cm}^{2}$. The patterned $\mathrm{Al}$ cathode had an isolated island type geometry to exclude the overestimation of the photocurrent as it commonly occurs when a cross-bar type geometry is

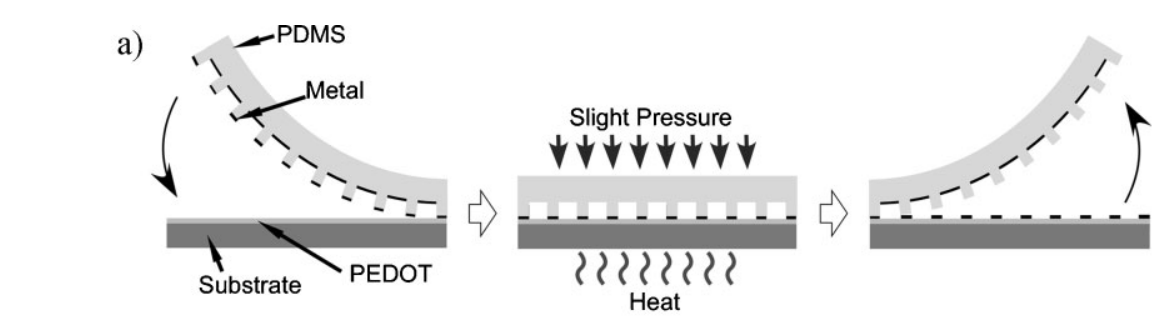

b)

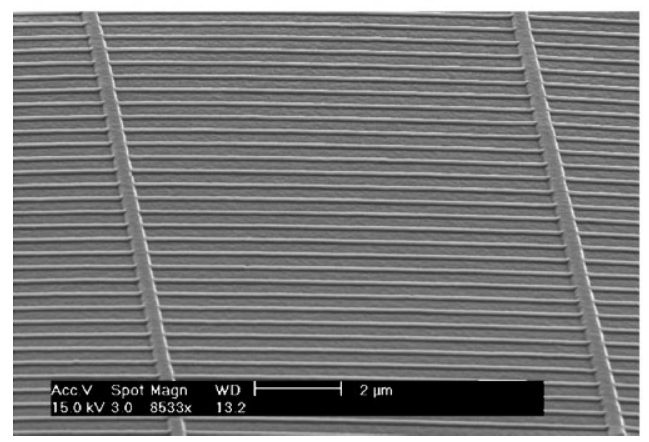

c)

Figure 4. a) Schematic of the fabrication of nanopatterned metal electrodes on PEDOT:PSS-coated glass substrates using a flexible PDMS stamp; b) SEM image of the PDMS stamp with a $40 \mathrm{~nm}$-thick Cu layer deposited on top; c) SEM image of the Cu electrode after transfer to the PEDOT:PSS-coated substrate. The inset shows the cross-section of the transferred metal electrode. 


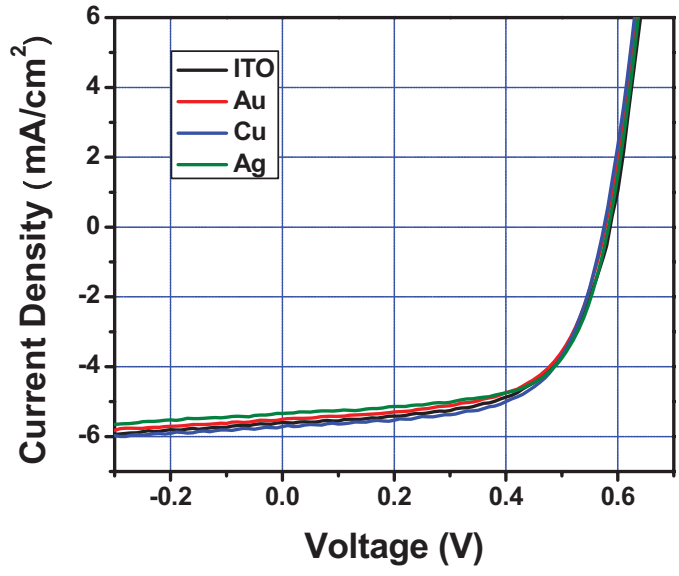

Figure 5. Current density versus voltage characteristics of organic solar cells (OSCs) fabricated using different electrodes, nanopatterned $\mathrm{Au}, \mathrm{Cu}$, and $\mathrm{Ag}$ as well as conventional ITO electrodes. (Intensity: AM 1.5C $100 \mathrm{~mW} \cdot \mathrm{cm}^{-2}$ ).

used. ${ }^{[25,26]}$ Current versus voltage characteristics were measured in air by illuminating the OSC devices with AM $1.5 \mathrm{G}$ simulated sun light (Oriel Solar Simulation, $100 \mathrm{~mW} \cdot \mathrm{cm}^{-2}$ ). As shown in Figure 5, the current versus voltage characteristics of the solar cells with the nanopatterned transparent metal electrodes and the ITO electrode are very similar to each other, indicating that such electrodes are interchangeable. All devices showed a power conversion efficiency of $\sim 2 \%$. Detailed device characteristics are summarized in Table 1 . Note that the power conversion efficiencies are lower than the ones reported in the literature, because of all our devices being fabricated and tested in an ambient environment with no special control of oxygen and/or moisture. However, PEDOT and the blend material were spin-coated and baked in a nitrogen glove box.

Although the nanopatterned metal electrodes are $8-18 \%$ less transparent than ITO at the absorption band of P3HT:PCBM $(\sim 500 \mathrm{~nm})$, very similar photocurrents were generated upon equivalent intensity light illumination. We believe that light trapping effects induced by the grating structure of the high transparency metal electrodes, ${ }^{[17-20]}$ compensate for their lower transmittance, which results in a similar absorption of light through the P3HT:PCBM blend layer. Interestingly, the device comprising the $\mathrm{Cu}$ electrode showed a slightly increased photocurrent and power conversion efficiency $(2.06 \%)$ as compared to those with the $\mathrm{Au}$ or ITO electrodes. From Figure 3 it can be seen that the

Table 1. Device characteristics of solar cells fabricated using nanopatterned $\mathrm{Au}, \mathrm{Cu}$, and $\mathrm{Ag}$ and conventional ITO electrodes (Intensity: AM 1.5C $100 \mathrm{~mW} \cdot \mathrm{cm}^{-2}$.

\begin{tabular}{lcccc}
\hline Transparent Electrode & $J_{\text {sc }}\left[\mathrm{mA} \cdot \mathrm{cm}^{-2}\right]$ & $V_{\text {oc }}[\mathrm{V}]$ & $\mathrm{FF}[\%]$ & Efficiency [\%] \\
\hline ITO & 5.59 & 0.59 & 0.61 & 2.00 \\
$\mathrm{Au}$ & 5.50 & 0.57 & 0.62 & 1.96 \\
$\mathrm{Cu}$ & 5.71 & 0.57 & 0.63 & 2.06 \\
$\mathrm{Ag}$ & 5.34 & 0.58 & 0.65 & 2.00 \\
\hline
\end{tabular}

transmittance of the $\mathrm{Cu}$ electrode is similar to that of the $\mathrm{Au}$ electrode, which in principle should produce the same photocurrent. The only difference between the $\mathrm{Au}$ and $\mathrm{Cu}$ electrode containing devices is the interface between metal electrode and PEDOT:PSS layer. We believe that the interface between $\mathrm{Cu}$ and PEDOT:PSS is more efficient in collecting holes than the one between Au and PEDOT:PSS due to the following reason: An anode with a lower work function than PEDOT:PSS is preferential for the collection of photogenerated holes The fact that $\mathrm{Au}$ has a work function of about $5.22 \mathrm{eV}$, which is slightly higher than that of PEDOT:PSS, is likely to impede with the hole transfer from the PEDOT:PSS layer to the Au electrode. On the other hand, $\mathrm{Cu}$ has a lower work function $(\sim 4.65 \mathrm{eV})$ than Au which facilitates better hole collection. The effect of a reduced internal electric field on the photocurrent, ${ }^{[27]}$ is negligible in devices with transparent metal electrodes because the metal electrodes occupy only $13 \%$ of the total anode area. Therefore, we expect improvements in device performance through efficient hole collection by using metals with lower work functions than PEDOT:PSS. This may also explain why the performance of the device with the $\mathrm{Ag}$ electrode, which has a lower transmittance than the other transparent electrodes, is comparable to that of the other devices. However, differences in transmittance between different metal electrodes could be another factor contributing to device performance.

Even though the hole collection efficiency can be increased by using metals with lower work functions, the metal nanograting only covers a small fraction of the anode region and the photo-generated holes still have to be transported through the continuous PEDOT:PSS layer to the metal-wire electrode to be measured. Therefore, it is important to evaluate if the conductive PEDOT:PSS layer can deliver the holes efficiently to the metal electrode. In order to quantify this, one can estimate the voltage drop in the PEDOT layer along the distance the holes have to travel. By employing a sheet resistance of $150,000 \Omega \cdot \mathrm{sq}^{-1}$ for the $100-\mathrm{nm}$ thick PEDOT:PSS layer and $350 \mathrm{~nm}$ as the maximum distance the holes have to travel, we estimated that the voltage drop is only in the order of $\mu \mathrm{V}$ for each period, even when we considered a high efficiency device that delivers a photocurrent of $10 \mathrm{~mA} \cdot \mathrm{cm}^{-2}$. Moreover, the voltage drop over the entire active area of the device (i.e., $\sim 0.1 \mathrm{~cm}^{2}$ ) is only several $\mathrm{mV}$. From these considerations, we can conclude that transparent metal-wire electrodes can effectively be treated as uniform films for the purpose of photocurrent collection. Based on this analysis, a simple design rule that correlates the optimum period of the nanopatterned metal electrodes with the sheet resistance of the PEDOT:PSS was derived, and is shown in Figure 6. Assuming a device area of approximately $0.1 \mathrm{~cm}^{2}$ and allowing for a voltage drop of $10 \mathrm{mV}$ over the whole PEDOT:PSS area, the appropriate grating period of the nanopatterned metal electrode at a certain sheet resistance of PEDOT:PSS can be found in the shaded area. The darker the region in the shaded area, the better the OSC performance will be. Hereby the sole assumption being made is that the optical 


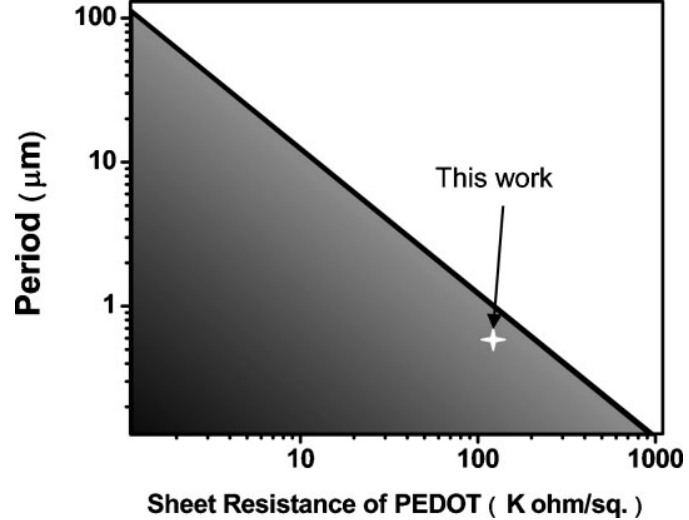

Figure 6. Calculated grating period of the nanopatterned transparent OSC electrodes as a function of the sheet resistance of PEDOT:PSS. Choosing a metal grating period and a PEDOT sheet resistance within the shaded region will lead to negligible loss of photocurrent. The star in the shaded region indicates the parameters corresponding to this work.

transmittance of the metal electrode needs to be comparable to that of an ITO electrode. This means that the line-width of the grating is about $10 \%$ of the period. The star in the shaded region indicates the parameters used in this work. Outside the shaded area transparent metal electrodes will still be able to collect generated photocurrent but with reduced efficiency due to an increased voltage drop in the PEDOT:PSS layer. As a proof, we also fabricated a device with an imprinted transparent $\mathrm{Au}$ electrode with a $10 \mu \mathrm{m}$ period. The short circuit current of the device was $80 \%$ of the ITO control device which led to a $20 \%$ lower power conversion efficiency. This result is supported by the fact that a $10 \mu \mathrm{m}$ period is outside the shaded region in Figure 6.

In summary, we demonstrated that various metal-wire electrodes fabricated by nanoimprint lithography can replace conventional ITO electrodes in solar cell fabrication. The nanopatterned metal electrodes showed high optical transmittance in the visible range as well as high electrical conductivity. Organic solar cells containing nanopatterned metal electrodes of optimum geometry showed essentially the same performance as devices made with high quality ITO electrodes. In addition, the effect of the work function of the nanopatterned electrode on the device performance was analyzed. It was found that metal electrodes with a lower work function than PEDOT:PSS facilitated better charge transfer from the PEDOT:PSS layer to the metal electrode leading to increased FFs and higher power efficiencies. We also demonstrated the fabrication of nanopatterned electrodes by using a flexible stamp and a metal transfer process, which shows the feasibility of extending their fabrication to a roll-to-roll process. The use of very inexpensive $\mathrm{Cu}$ material as transparent electrodes and a possible roll-to-roll fabrication could help to realize low cost, large area organic solar cells.

\section{Experimental}

Mold Fabrication: An original $700 \mathrm{~nm}$-period $\mathrm{SiO}_{2}$ grating mold with a depth of $500 \mathrm{~nm}$ and a duty cycle of $50 \%$ was etched using buffered HF solution (Transene, Buffered HF improved) for $80 \mathrm{~s}$, which resulted in a line-width of $\sim 70 \mathrm{~nm}$, as shown in Figure 1 . The $\mathrm{SiO}_{2}$ etch rate was about $100 \mathrm{~nm} \cdot \mathrm{min}^{-1}$. The second grating mold with a period of $10 \mu \mathrm{m}$ and a line-width of $850 \mathrm{~nm}$, which was created by conventional photolithography, was also etched with buffered HF solution to reduce the line-width to $400 \mathrm{~nm}$. Using these two molds, a new mold with a rectangular mesh grid was fabricated by applying nanoimprint lithography (NIL) twice. A Nanonex NX2000 naonoimprinter (Princeton, NJ) was used for the NIL process. The $10 \mu \mathrm{m}$ period mold was first used to imprint a $140 \mathrm{~nm}$ thick MRI-8020 resist (Microresist Technology $\mathrm{GmbH}$ ), spin-coated on a $\mathrm{SiO}_{2}$ substrate, at a pressure of $600 \mathrm{psi}(4.14 \mathrm{MPa})$ and a temperature of $180^{\circ} \mathrm{C}$ for $5 \mathrm{~min}$. After cooling and demolding, an anisotropic oxygen plasma etching was used to remove residual resist from the imprinted areas. After this an additional $20 \mathrm{~nm}$-thick $\mathrm{Cr}$ layer was deposited and used as $\mathrm{SiO}_{2}$ etching mask after a lift-off in acetone. The grating mold with the $700 \mathrm{~nm}$ period was used for the second NIL, whereby the same procedure as above was followed except for the fact that the substrate was turned by $90^{\circ}$. The $\mathrm{SiO}_{2}$ was then plasma-etched for 8 min using trifluoromethane $\left(\mathrm{CHF}_{3}\right)$ and tetrafluoromethane $\left(\mathrm{CF}_{4}\right)$ at a flow rate of $15 \mathrm{sccm}$, a power of $100 \mathrm{~W}$, and a pressure of 15 mTorr $(1.99 \mathrm{~Pa})$, which resulted in a $140 \mathrm{~nm}$ deep grating. Treatment of the mold with an anti-sticking layer $(1 \mathrm{H}, 1 \mathrm{H}, 2 \mathrm{H}, 2 \mathrm{H}$-perfluorodecyl trichlorosilane) after $\mathrm{Cr}$ removal completed the fabrication of the mold with the rectangular shaped grid pattern.

Fabrication of Metal Electrodes on PEDOT Layers: A nanoimprinted resist template was first prepared by NIL using the mold shown in Figure 2a. For the fabrication of the PDMS stamp, high modulus PDMS was drop casted and cured at $70^{\circ} \mathrm{C}$ for 5 min producing a $\sim 100 \mu \mathrm{m}$-thick PDMS layer on the resist. Such high modulus PDMS is necessary for the replication of nanoscale features and to avoid collapsing of the pattern. The detailed composition of this fast curing and high modulus PDMS can be found in [23]. Commercially available PDMS, Sylgard 184 , was then drop casted on top and cured at $70^{\circ} \mathrm{C}$ for $2 \mathrm{~h}$ to support the thin layer of high modulus PDMS. After removing the PDMS stamp from the resist template a $40 \mathrm{~nm}$ thick $\mathrm{Cu}$ layer and a $2 \mathrm{~nm}$ thick Ti layer were sequentially deposited on the PDMS stamp by electron-beam evaporation at a rate of $2 \AA \cdot \mathrm{s}^{-1}$. The $40 \mathrm{~nm}$-thick $\mathrm{Cu}$ layer was then transferred to a PEDOT:PSS layer, which was spincoated on a PET or $\mathrm{SiO}_{2}$ substrate, at a pressure of $10 \mathrm{psi}(68.9 \mathrm{kPa})$ and a temperature of $80^{\circ} \mathrm{C}$ for $1 \mathrm{~min} .0 .2 \mathrm{wt} \%$ Silquest 187 and glycerol were added to the PEDOT:PSS mixture to increase wetting of PEDOT:PSS to the substrate, and prevent the solvent from evaporating, respectively.

Fabrication of Organic Solar Cells: Transparent metal electrodes $(\mathrm{Au}, \mathrm{Cu}$ and $\mathrm{Ag}$ ) and ITO electrodes on glass were cleaned in acetone and isopropyl alcohol (IPA) under sonication for $30 \mathrm{~min}$ each. ITO coated glass was purchased from Delta Technologies, LTD. Cleaned substrates were then transferred to a $\mathrm{N}_{2}$ purged glove box and filtered PEDOT:PSS, which was purchased from H.C. Starck, was spin-coated onto the metal electrodes at $3000 \mathrm{~min}^{-1}$ for $30 \mathrm{~s}$, producing a $95 \mathrm{~nm}$ thick layer which was subsequently baked at $120^{\circ} \mathrm{C}$ for $15 \mathrm{~min}$. In order to increase the wetting to the substrate and control the PEDOT:PSS thickness, $0.2 \mathrm{wt} \%$ Silquest 187 and $30 \mathrm{wt} \%$ IPA were added to the PEDOT. The light absorbing material, a blend of P3HT and PCBM (1:1 ratio by weight) dissolved in chlorobenzene and filtered, was spincoated onto the PEDOT:PSS layer at $1000 \mathrm{~min}^{-1}$ for $30 \mathrm{~s}$, which gave a $105 \mathrm{~nm}$ thick layer, that was annealed at $130^{\circ} \mathrm{C}$ for $20 \mathrm{~min}$. P3HT and PCBM were purchased from Rieke Metals Ltd. and American Dye source, respectively. After cooling to room temperature, samples were brought from the $\mathrm{N}_{2}$ glove box to an evaporation chamber. Thermal evaporation of a $1 \mathrm{~nm}$-thick $\mathrm{LiF}$ layer followed by a $70-\mathrm{nm}$ thick $\mathrm{Al}$ layer through a shadow mask completed the organic solar cell fabrication. 
[1] K. M. Coakley, M. D. McGehee, Chem. Mater. 2004, 16, 4533.

[2] H. Hoppe, N. S. Sariciftci, J. Mater. Res. 2004, 19, 1924.

[3] J. Y. Kim, K. Lee, N. E. Coates, D. Moses, T.-Q. Nguyen, M. Dante, A. J. Heeger, Science 2007, 317, 222.

[4] G. Li, V. Shrotriya, J. Huang, Y. Yao, T. Moriarty, K. Emery, Y. Yang, Nature Mater. 2005, 4, 864.

[5] G. Li, V. Shrotriya, Y. Yao, Y. Yang, J. Appl. Phys. 2005, 98 , 043704.

[6] W. Ma, C. Yang, X. Gong, K. Lee, A. J. Heeger, Adv. Funct. Mater. 2005, 15, 1617.

[7] W. S. Jahang, A. H. Francis, H. Moon, J. I. Nanos, M. D. Curtis, Appl. Phys. Lett. 2006, 88, 093504.

[8] Z. Chen, B. Cotterel, W. Wang, E. Guenther, S.-J. Chua, Thin Solid Films 2001, 394, 202.

[9] M. W. Rowell, M. A. Topinka, M. D. McGehee, H.-J. Prall, G. Dennler, N. S. Sariciftci, L. Hu, G. Gruner, Appl. Phys. Lett. 2006, 88, 233506.

[10] A. J. Miller, R. A. Hatton, G. Y. Chen, S. Ravi, P. Silva, Appl. Phys. Lett. 2007, 90, 023105.

[11] J. van de Lagemaat, T. M. Barnes, G. Rumbles, S. E. Shaheen, T. J. Coutts, C. Weeks, I. Levitsky, J. Peltola, P. Glatkowski, Appl. Phys. Lett. 2006, 88, 233503.

[12] A. J. Miller, R. A. Hatton, S. Ravi, P. Silva, Appl. Phys. Lett. 2006, 89, 133117.
[13] K. Tvingstedt, O. Inganäs, Adv. Mater. 2007, 19, 2893.

[14] J.-Y. Lee, S. T. Connor, Y. Cui, P. Peumans, Nano Lett. 2008, 8, 689

[15] L. J. Guo, Adv. Mater. 2007, 19, 495.

[16] M.-G. Kang, L. J. Guo, Adv. Mater. 2007, 19, 1391.

[17] L. S. Roman, O. Inganäs, T. Granlund, T. Nyberg, M. Svensson, M. R. Andersson, J. C. Hummelen, Adv. Mater. 2000, 12, 189.

[18] S.-I. Na, S.-S. Kim, S.-S. Kwon, J. Jo, J. Kim, T. Lee, D.-Y. Kim, Appl Phys. Lett. 2007, 91, 173509.

[19] C. Cocoyer, L. Rocha, L. Sicot, B. Geffroy, R. de Bettignies, C. Sentein, C. Fiorini-Debuisschert, P. Raimond, Appl. Phys. Lett. 2006, 88, 133108.

[20] M.-S. Kim, J.-S. Kim, J. C. Cho, M. Shtein, L. J. Guo, J. Kim, Appl. Phys. Lett. 2007, 90, 123113.

[21] S. Ahn, L. J. Guo, Adv. Mater. 2008, 20, 2044.

[22] N. D. Lang, W. Kohn, Phys. Rev. B 1971, 3, 1215.

[23] C. Pina-Hernandez, J.-S. Kim, L. J. Guo, P.-F. Fu, Adv. Mater. 2007, $19,1222$.

[24] M.-G. Kang, L. J. Guo, J. Vac. Sci. Technol, B 2007, 25, 2637.

[25] A. Cravino, P. Schilinsky, C. J. Brabec, Adv. Funct. Mater. 2007, 17, 3906.

[26] M.-S. Kim, M.-G. Kang, L. J. Guo, J. Kim, Appl. Phys. Lett. 2008, 92, 133301.

[27] V. D. Mihailetchi, L. J. A. Koster, P. W. M. Blom, Appl. Phys. Lett. 2004, 85,970 